\title{
The Interference Response of Reflected Transient Waves from Several Micro Plane Cracks
}

\author{
Kimihisa MIURA*, Naoto SHOUJ**, Gui-Rong LIU*** and Tadashi OHYOSHI* \\ *Department of Mechanical Engineering Faculty of Engineering and Resource Science, \\ Akita University 1-1, Tegata-Gakuen-Cho, Akita, 010-8502, JAPAN \\ **Hitachi Unisia. Ltd., 1-2, Shimo-Goshono, Yokote, 013-8592, JAPAN \\ ***Department of Mechanical Engineering, National University of Singapore, Singapore \\ E-mail:kmiura@ipc.akita-u.ac.jp
}

\begin{abstract}
On the concave mirror which has the curvature of the paraboloid, the plane transient wave impinging to the mirror perpendicularly is reflected and wave energy is collected in one focus. Therefore, it is widely used as a mirror for kindling a torch in the Olympics and a parabolic antenna for the radio wave reception. This phenomenon can be easily explained by the ray theory and it can be freely applied for the steady incidence and transient incidence of the wave. But, when not only the concentration point of the wave energy but also the interference response of the reflected waves which spreads in various directions from the specific boundary are examined, the exact analysis of the reflection response is required and it can not be easily grasped. If the position of an energy-minimum point by the complicated interference of reflected transient waves is detected, it becomes engineeringly a very important knowledge from the viewpoint of the material protection. In this study, the interference response of SH reflected transient waves made by the reflection system composed of several micro plane cracks is examined with the exact analysis, and the numerical results are shown using 3-D graphic representations.
\end{abstract}

Key Words : Interference Response, SH Transient Analysis, Several Micro Plane Cracks

\section{INTRODUCTION}

The analysis of transient wave propagation in the elastic medium is applied to the ultrasonic detection ${ }^{1)}$ of structural material and the lithotripsy ${ }^{2)}$. If we seek only the concentration point of the wave energy by transient loading, we can easily calculate it by the ray theory and freely apply it for the steady or transient incidence of wave to the material. But, if we investigate the interference response of the reflected wave which spreads in various directions from the specific boundary, the exact analysis of the reflected response is required and it can not be easily grasped. If the position of an energy-minimum point by the complicated interference of the reflected response for the transient wave incidence is detected, it becomes engineeringly a very important knowledge from the viewpoint of the material protection. Recently, the application of TRM (The Time Reversal Mirror) to the medical and engineering fields is variously being $\operatorname{tried}^{3 \sim 4)}$. In this study, as an example for examining the response of the wave field by the interference of several transient waves, the interference response between SH incident transient wave and the reflected wavefronts made by the reflection system composed of several micro plane cracks is examined with the exact analysis.

\section{ANALYSIS}

\subsection{Transient reflection response}

We consider an antiplane deformation problem which $\mathrm{SH}$ transient load acts on the free surface of a homogeneous half-space. Our main purpose is to examine the interference of a few transient waves. As one example, several micro plane cracks are placed in the half-space in order to concentrate each reflected transient wave energy in one focusing point. We assume that each micro plane crack reflects only one incident wave ray, and we adjust the angle of the plane crack so that the largest reflection response will appear just on the focusing point. If the response for one crack is analyzed, we can calculate for several cracks by the superposition of the solution because of the linearity of the response. Then, we consider the problem like Figure 1. The crack exists at the angle $\phi$ for the free surface and we consider the $X^{-}$ $Y$ local rotated coordinate which the crack coordinate (the center of the crack $\left(x_{c}, y_{c}\right)$ becomes the origin of coordinate. When the wavefront yielded by the impact on the free surface of half-space impinge the micro crack, we can consider that the secondary transient load simultaneously acts on the crack surface. Since we have already obtained the exact solution of the transient response for half-space ${ }^{5)}$, it is verified that the response of the arbitrary point becomes almost steady state by about 2 times of the time which the wavefront reaches that point ${ }^{6}$. Then, we can easily estimate the 
secondary transient load only if the crack coordinate $\left(x_{c}, y_{c}\right)$ and the angle $\phi$ are decided. We assume that the new secondary transient load acts on the virtual plane boundary which crack surface is expanded. Then, applying the analytic continuation of two half-spaces (the reflection region and the diffraction region from the crack surface), the transient response of the infinite solid is obtained from two responses for the half-space. We are going to call this response of the infinite solid, the reflection response from the crack. Since the wavefront reaches the crack at the dimensionless time $\tau_{c}=c t / \sqrt{x_{c}^{2}+y_{c}^{2}}$, total response becomes no crack response before at $\tau=\tau_{c}$, and becomes the superposition of the initial response and the reflection response after at $\tau=\tau_{c}$, where, the nondemensional time $\tau$ is

$$
\tau=c t / x
$$

and $c$ is SH wave phase velocity. Therefore if we represent no crack response is $f_{1}(x, y, \tau)$ and the reflection response from the crack is $f_{2}(x, y, \tau)$, the response of total wave field $f(x, y, \tau)$ becomes as following

$$
\begin{aligned}
& f(x, y, \tau)=f_{1}(x, y, \tau) \quad \text { at } \tau<\tau_{c} \\
& f(x, y, \tau)=f_{1}(x, y, \tau)+f_{2}\left(x, y, \tau-\tau_{c}\right) \quad \text { at } \tau \geqq \tau_{c}
\end{aligned}
$$

The boundary condition on the free surface of half-space and crack given by respectively

$$
\begin{aligned}
& \left.\tau_{y z}\right|_{y=0}=Q \delta(x) H(\tau) \\
& \left.\tau_{y z}\right|_{\substack{x=x_{c} \\
y=y_{c}}}=Q_{y} \delta\left(x-x_{c}\right) \delta\left(y-y_{c}\right) H\left(\tau-\tau_{c}\right) \\
& \tau_{\substack{\left.x z\right|_{x=x_{c}} \\
y=y_{c}}}=Q_{y} \delta\left(x-x_{c}\right) \delta\left(y-y_{c}\right) H\left(\tau-\tau_{c}\right)
\end{aligned}
$$

where, $Q$ is the force amplitude, $\delta(x)$ is the Dirac's delta function, $H(\tau)$ is the Heaviside's step function and $Q_{y}, Q_{x}$ are the following transient stress values getting the steady state $\left(\tau=2 \tau_{c}\right)$ on the crack position

$$
\begin{aligned}
& Q_{y}=\tau_{y z}\left(x_{c}, y_{c}, 2 \tau_{c}\right)=Q \cos \theta / \pi \sqrt{x_{c}^{2}+y_{c}^{2}} \\
& Q_{x}=\tau_{x z}\left(x_{c}, y_{c}, 2 \tau_{c}\right)=Q \sin \theta / \pi \sqrt{x_{c}^{2}+y_{c}^{2}}
\end{aligned}
$$

In the origin of the $X-Y$ local rotated coordinate, Eqs. (5) and (6) are replaced as following

$$
\left.\tau_{Y Z}\right|_{Y=0}=Q_{y} \delta(X) H\left(\tau-\tau_{c}\right)
$$

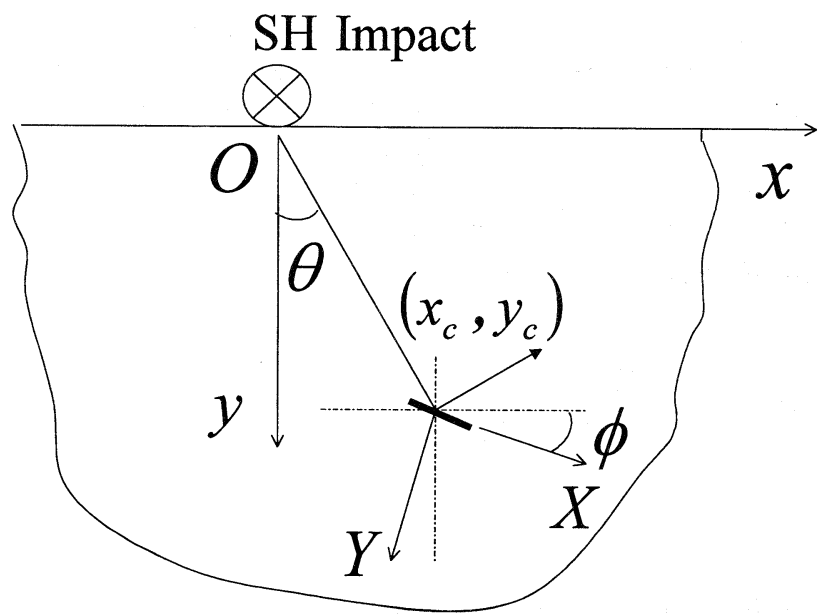

Figure 1 Reflection of SH transient waves from a micro plane crack

$$
\left.\tau_{X Z}\right|_{Y=0}=Q_{x} \delta(X) H\left(\tau-\tau_{c}\right)
$$

Then, the response of all wave field is

$$
\begin{aligned}
& {\left[\begin{array}{l}
\tau_{y z} \\
\tau_{x z}
\end{array}\right]=\left[\begin{array}{ll}
\tau_{y z}(x, y, \tau) \\
\tau_{x z}(x, y, \tau)
\end{array}\right]_{1}} \\
& +\left[\begin{array}{ll}
\cos \phi & \sin \phi \\
-\sin \phi & \cos \phi
\end{array}\right]\left[\begin{array}{l}
\tau_{Y z}\left(x_{r}, y_{r}, \tau-\tau_{c}\right) \\
\tau_{X z}\left(x_{r}, y_{r}, \tau-\tau_{c}\right)
\end{array}\right]
\end{aligned}
$$

where

$$
\left[\begin{array}{l}
x_{r} \\
y_{r}
\end{array}\right]=\left[\begin{array}{rr}
\cos \phi & -\sin \phi \\
\sin \phi & \cos \phi
\end{array}\right]\left[\begin{array}{l}
X \\
Y
\end{array}\right]
$$

and for the observation coordinate $(x, y)$

$$
\left[\begin{array}{l}
x \\
y
\end{array}\right]=\left[\begin{array}{l}
x_{r} \\
y_{r}
\end{array}\right]+\left[\begin{array}{l}
x_{0} \\
y_{0}
\end{array}\right]
$$

$\left(x_{0}, y_{0}\right)$ is the value calculated from the crack position, the calculation mesh point and the elapsed time. In the case which $m$ pieces of the plane crack are considered, $\left(x_{r}, y_{r}\right),\left(x_{0}, y_{0}\right), \phi$ become $\left(x_{r m}, y_{r m}\right),\left(x_{0 m}, y_{0 m}\right), \phi_{m}$ and Eq. (11) become as following

$$
\begin{aligned}
& {\left[\begin{array}{l}
\tau_{y z} \\
\tau_{x z}
\end{array}\right]=\left[\begin{array}{l}
\tau_{y z}(x, y, \tau) \\
\tau_{x z}(x, y, \tau)
\end{array}\right]_{1}} \\
& +\sum_{i=1}^{m}\left[\begin{array}{ll}
\cos \phi_{i} & \sin \phi_{i} \\
-\sin \phi_{i} & \cos \phi_{i}
\end{array}\right]\left[\begin{array}{c}
\tau_{Y z i}\left(x_{r i}, y_{r i}, \tau-\tau_{c i}\right) \\
\tau_{X z i}\left(x_{r i}, y_{r i}, \tau-\tau_{c i}\right)
\end{array}\right]
\end{aligned}
$$

The strain energy $U$ is

$$
U=\frac{1}{4 \mu}\left(\tau_{y z}^{2}+\tau_{x z}^{2}\right)
$$

\subsection{SH transient analysis of homogeneous half-space}

When there is no crack the equation of motion of the SH mode becomes as following

$$
\frac{\partial^{2} w}{\partial x^{2}}+\frac{\partial^{2} w}{\partial y^{2}}=\frac{1}{c^{2}} \frac{\partial^{2} w}{\partial t^{2}}
$$

where

$$
c=\sqrt{\mu / \rho}
$$

$w$ is $z$ direction displacement, $\mu$ is the modulus of transverse elasticity and $\rho$ is the mass density of the material. The initial conditions are

$$
\left.w\right|_{t=0}=\left.\dot{w}\right|_{t=0}=0
$$

The integral transforms will be defined as following for the Fourier integral transform

$$
\begin{aligned}
& \mathscr{F}[w] \equiv \bar{w}(s \xi)=\int_{-\infty}^{\infty} w(x) \mathrm{e}^{-\mathrm{i} \xi \mathrm{x}} d x \\
& \mathscr{F}^{-1}[\bar{w}] \equiv w(x)=\frac{1}{2 \pi} \int_{-\infty}^{\infty} \bar{w}(s \xi) \mathrm{e}^{-i \mathrm{~s} \xi \mathrm{x}} d(s \xi)
\end{aligned}
$$

for the Laplace integral transform

$$
\begin{aligned}
& \mathscr{L}^{-1}[w] \equiv w^{*}(s)=\int_{0}^{\infty} w(t) \mathrm{e}^{-s t} d t \\
& \mathscr{L}^{-1}\left[w^{*}\right] \equiv w(t)=\frac{1}{2 \pi i} \int_{\mathrm{Br}} w^{*}(s) \mathrm{e}^{\mathrm{st}} d s
\end{aligned}
$$

where, $\mathrm{Br}$ of $\mathrm{Eq}$. (22) stands for the Bromwhich-Wagner integration. Considering the boundary condition (4) and applying 
the Fourier Laplace transforms to Eq. (16), we obtain the transformed solution. Furthermore, applying the Cagniard method to evaluate exactly the double inversion, we finally obtain the following exact solutions for the stress components.

$$
\begin{aligned}
& \tau_{y z}=\frac{Q}{\pi} H(t-v R) \frac{y t}{R^{2} \sqrt{t^{2}-v^{2} R^{2}}} \\
& \tau_{x z}=\frac{Q}{2 \pi} H(t-v R) \cdot \operatorname{Im}\left[\frac{\xi_{+}}{\sqrt{\xi^{2}+v^{2}}} \frac{\partial \xi_{+}}{\partial t}-\frac{\xi_{-}}{\sqrt{\xi^{2}-v^{2}}} \frac{\partial \xi_{-}}{\partial t}\right]
\end{aligned}
$$

where

$$
\begin{aligned}
& v=1 / c \\
& \xi_{ \pm}=\frac{ \pm y \sqrt{t^{2}+v^{2} R^{2}}+i x t}{R^{2}} \\
& R=\sqrt{x^{2}+y^{2}}
\end{aligned}
$$

Similarly, the exact solutions of the transient reflection analysis from the $i$ th crack are obtained using the boundary conditions (9), (10) as following

$$
\begin{aligned}
& \tau_{Y Z i}=\tau_{Y Z i 1}+\tau_{Y Z i 2} \\
& \tau_{X Z i}=\tau_{X Z i 1}+\tau_{X Z i 2}
\end{aligned}
$$

where

$$
\begin{aligned}
\tau_{Y Z i 1} & =\frac{Q_{y i}}{\pi} \mathrm{H}\left(t_{i}-v R_{i}\right) \frac{Y_{i} t_{i}}{R_{i}^{2} \sqrt{t_{i}^{2}-v^{2} R_{i}^{2}}} \\
\tau_{Y Z i 2} & =\frac{Q_{y i}}{2 \pi} \mathrm{H}\left(t_{i}-v R_{i}\right) \cdot \operatorname{Im}\left[\frac{\sqrt{\xi_{i+}^{2}+v^{2}}}{\xi_{i+}} \frac{\partial \xi_{i+}}{\partial t_{i}}-\frac{\sqrt{\xi_{i-}^{2}+v^{2}}}{\xi_{i-}} \frac{\partial \xi_{i-}}{\partial t_{i}}\right]
\end{aligned}
$$

$\tau_{X Z i 1}=\frac{Q_{x i}}{2 \pi} \mathrm{H}\left(t_{i}-v R_{i}\right) \cdot \operatorname{Im}\left[\frac{\xi_{i+}}{\sqrt{\xi_{i+}^{2}+v^{2}}} \frac{\partial \xi_{i+}}{\partial t_{i}}-\frac{\xi_{i-}}{\sqrt{\xi_{i-}^{2}+v^{2}}} \frac{\partial \xi_{i-}}{\partial t_{i}}\right]$

$$
\tau_{X Z 2}=\frac{Q_{x i}}{\pi} \mathrm{H}\left(t_{i}-v R_{i}\right) \frac{Y_{i} t_{i}}{R_{i}^{2} \sqrt{t_{i}^{2}-v^{2} R_{i}^{2}}}
$$

$\xi_{i \pm}=\frac{ \pm Y_{i} \sqrt{t_{i}^{2}-v^{2} R_{i}^{2}}+i X_{i} t_{i}}{R_{i}^{2}}$

$$
\begin{aligned}
& R_{i}=\sqrt{X_{i}^{2}+Y_{i}^{2}} \\
& X_{i}=X-X_{c i} \\
& Y_{i}=Y-Y_{c i} \\
& t_{i}=t-t_{c i}
\end{aligned}
$$

\section{NUMERICAL CALCULATION}

First in the numerical calculation, we must determine the interference region between the crack position and the incident, reflected, diffractive waves for each crack. As the material is homogeneous and isotropic, and the dimensionless time Eq. (1) is introduced, the response of the wave propagation becomes the similar figure regardless of the material composition. Since the wavefront diffracted by one crack meets tangentially the incident wavefront as shown in Figure 2, for the total calculation mesh size $n$ which decides the interference region, the calculation mesh size, $M_{\tau c r}$, for the interference region radius, the calculation mesh size, $M_{\tau x}$, for $\tau_{x}$, the calculation mesh size, $M_{\tau y}$, for $\tau_{y}$ can be decided respectively as following

$$
M_{\tau c r}=\operatorname{int}\left(n \cdot \tau_{c r}\right) \quad \text { at } \quad 0 \leqq \tau \leqq \tau_{c r}
$$

$$
\begin{aligned}
& M_{\tau x}=\operatorname{int}\left(n \cdot \tau_{x}\right) \quad \text { at } \quad \tau=\tau_{x} \\
& M_{\tau y}=\operatorname{int}\left(n \cdot \tau_{y}\right) \quad \text { at } \quad \tau=\tau_{y}
\end{aligned}
$$

where

$$
\begin{aligned}
\tau_{c r} & =\tau_{1}-R_{c r} \\
\tau_{x} & =\tau_{1}+x_{c}-\tau_{c r} \\
\tau_{y} & =\sqrt{R_{c r}^{2}-x_{c}^{2}}-\tau_{c r} \\
R_{c r} & =\sqrt{x_{c}^{2}+y_{c}^{2}}
\end{aligned}
$$

The transient reflection response from several micro plane crack can be calculated by the superposition of each solution. As one numerical example, the effect of the reflection response from several cracks to all wave field is verified. Here, as shown in Figure 3 we consider that the reflection energy from 9 cracks will simultaneously concentrate at one focusing point. This focusing point coordinate is $\left(x_{f}, y_{f}\right)=(0,0.5)$ and 9 cracks is located on the specular surface of the imaged concave mirror with this point as one focus. Each crack angle was set so that the reflection energy becomes largest at the focusing point. Afterwards, we examine the transient reflection response. The positions and angles of 9 cracks are shown at Table 1 . Figure 4 shows the variation of strain energy for $x$ coordinate at the dimensionless time $\tau=1$ and $y$ coordinate $y=0.5$. Since the energy at the focusing point $(0$, 0.5 ) is too great, the vertical axis is made to be the value of

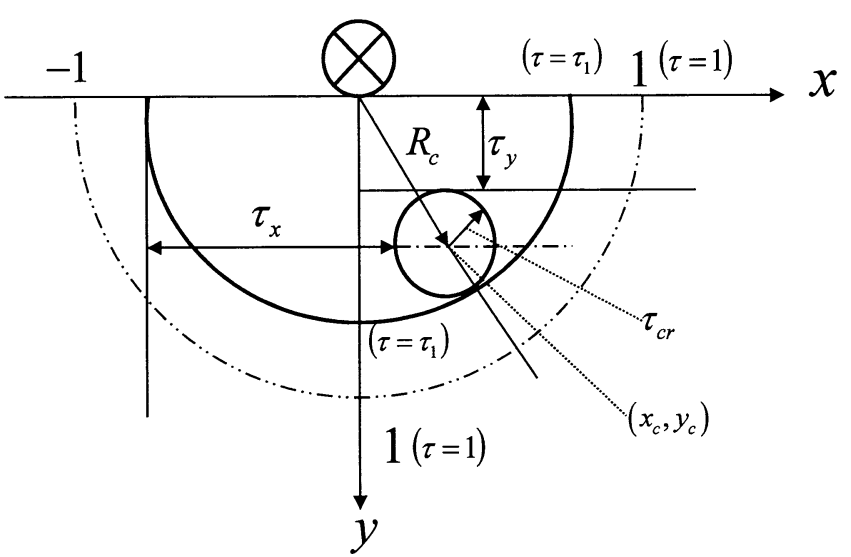

Figure 2 Geometric explanation of the reflected wavefront from one crack

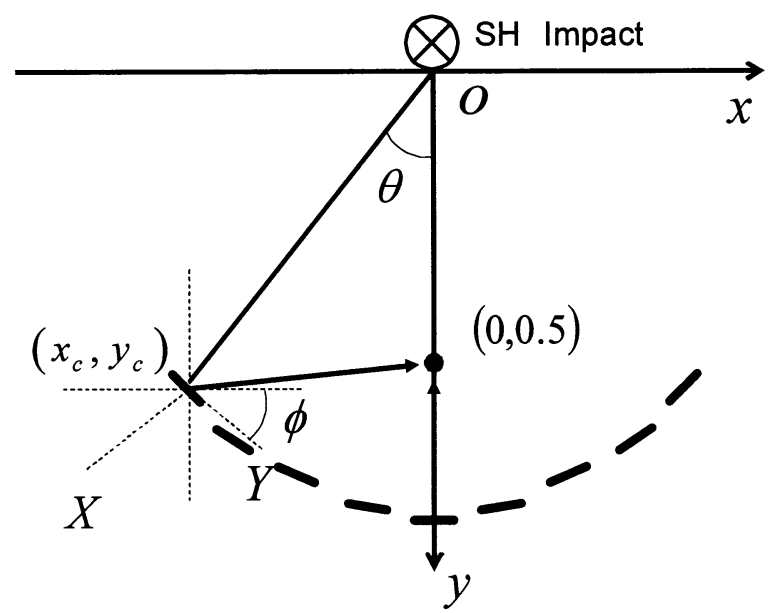

Figure 3 Reflection of the wavefront from 9 micro plane cracks to the focusing point $(0,0.5)$ 
Table 1 Crack coordinates and crack angles of 9 micro plane cracks

\begin{tabular}{|c|c|c|}
\hline Number & Crack position & Crack angle \\
\hline 1 & $(-0.34641016,0.55)$ & $\phi=56.9955084$ \\
\hline 2 & $(-0.30923292,0.6)$ & $\phi=49.6729156$ \\
\hline 3 & $(-0.25980762,0.65)$ & $\phi=40.8933946$ \\
\hline 4 & $(-0.18874586,0.7)$ & $\phi=29.2159716$ \\
\hline 5 & $(0.0,0.75)$ & $\phi=0.0$ \\
\hline 6 & $(0.18874586,0.7)$ & $\phi=150.7840284$ \\
\hline 7 & $(0.25980762,0.65)$ & $\phi=139.1066054$ \\
\hline 8 & $(0.30923292,0.6)$ & $\phi=130.3270844$ \\
\hline 9 & $(0.34641016,0.55)$ & $\phi=123.0044916$ \\
\hline
\end{tabular}

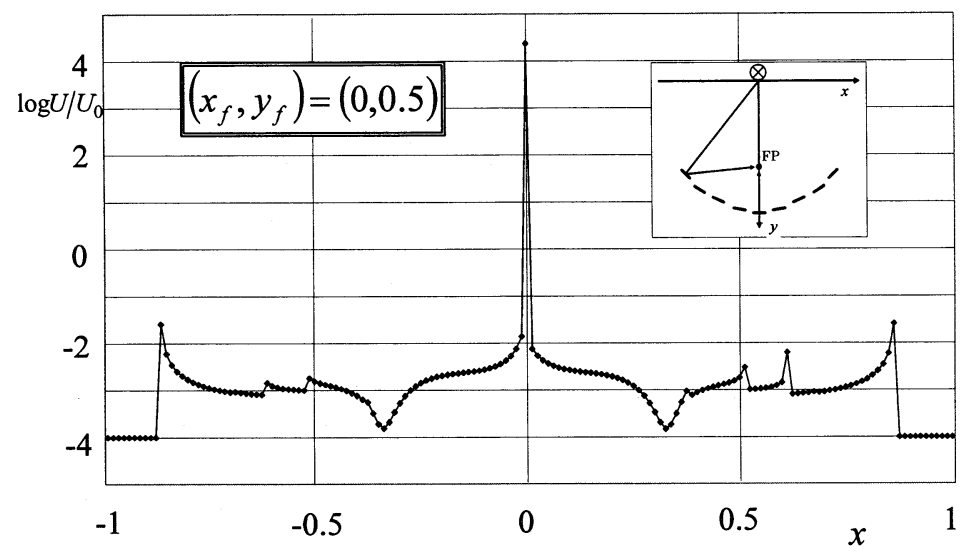

Figure 4 Variation of strain energy for $x$ coordinate at the dimensionless time $\tau=1$ and $y$ coordinate $y=0.5$

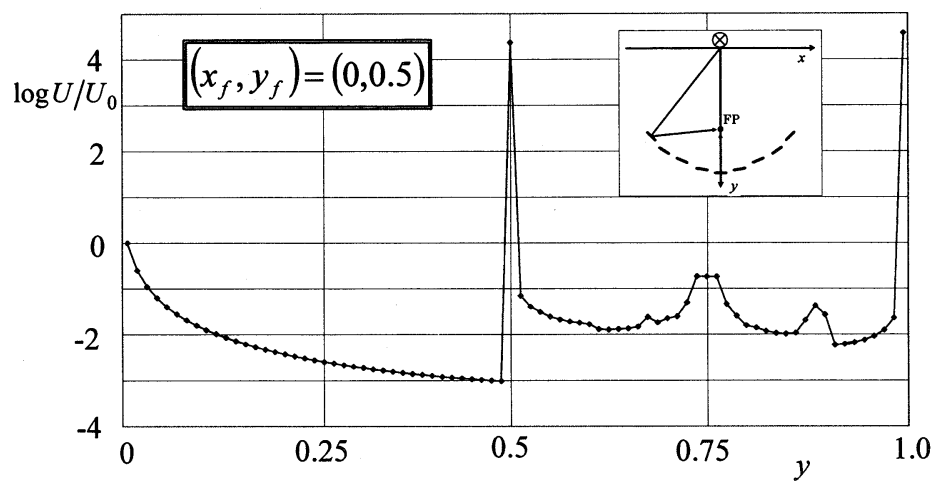

Figure 5 Variation of strain energy for $y$ coordinate at the dimensionless time $\tau=1$ and $x$ coordinate $x=0$ 

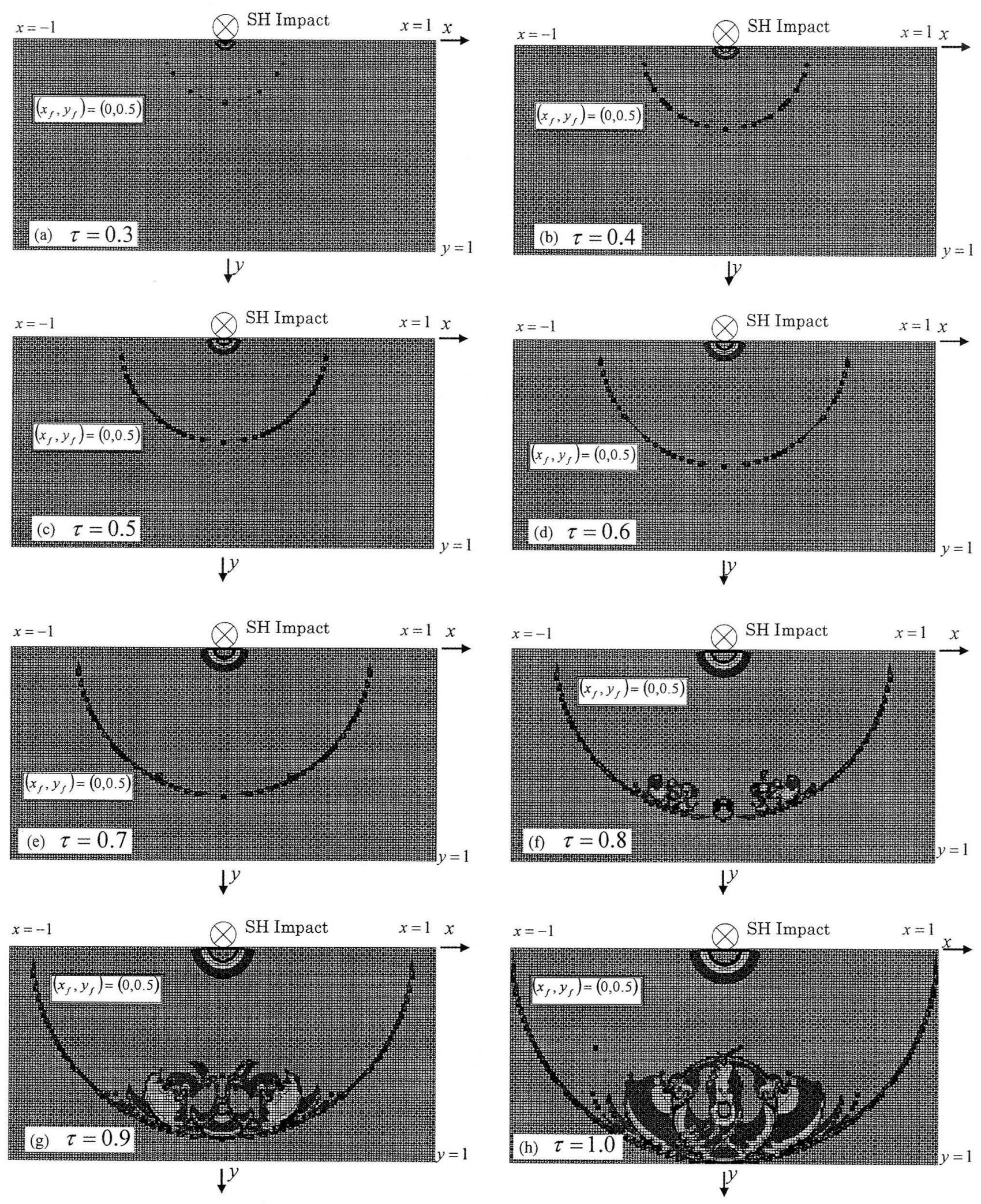

Figure 6 Contour mapping representations of the reflected strain energy from 9 micro plane cracks 
common logarithm $\log U / U_{0}$, where $U_{0}$ is the value of $U$ at the nearest calculation point from the wave source. In this figure, in the region where the wave front is not reaching, $U / U_{0}=0$ then $\log$ $U / U_{0}=-\infty$, therefore, we added 0.0001 to all $U$ value. Though the smallest energy in Figure 4 is shown by $\log U / U_{0}=-4$, it is not effective in the whole shape of the figure. Figure 5 shows the variation of the strain energy for $y$ coordinate at $x=0 . y$ is a region over 0.5 , we can see the interference of the reflected wave from several cracks and under 0.5 , it is shown that there is no reflected wave coming in from cracks. Figure 6 shows the contour mapping representations of the strain energy $U / U_{0}$ at the different times. From these figures we can get the process which the wavefront yielded on the surface of half-space at $\tau=0$ are reflected at each micro plane crack and are concentrated just on the focusing point $(0,0.5)$ at $\tau=1$. Figure 7 shows $3-\mathrm{D}$ graphic representation of the strain energy $U / U_{0}$ for the whole wave field. The reflection response should become symmetry. However, since the calculation mesh size has previously been decided, the superimposed position information error under the smallest calculation mesh width has appeared.

\section{CONCLUSION}

The reflection analysis of the transient wave from several micro plane cracks was carried out by using the exact solution analyzed by Cagniard method for SH transient response of half space. For the reflection response we considered the secondary transient load on one crack surface and got the solution for the infinite solid by the analytic continuation. Finally, in order to the linear analysis of this problem we could superimpose the reflection responses from several micro plane cracks. Therefore we could carry out the numerical calculation to which collected the transient reflection from 9 micro cracks at one focusing point, and could show the results with the 3-D graphic representation. On this analysis, even if increasing the crack number the load for the calculation does not increase especially. Therefore, making the crack arrange rectilinear, we will be able to approximate the plane boundary and also calculate the reflection response from the corner with various angles.

\section{References}

[1] Asraf, D.E. and Gustafsson, G, " Phenomenological Detectors for Crack Echo Families in Elastic Solids”, $J$. Acoust. Soc. Am., Vol.116, No.1, pp.379-388, (2004).

[2] Thomas, J.L., Wu, F. and Fink, M., "Time Reversal Focusing Applied to Lithotripsy", Ultrasonic Imaging, Vol.18, No.2, pp.106-121, (1996).

[3] Montaldo, G., Roux, P., Derode, A., Negreira, C. and Fink, M., "Generation of Very High Pressure Pulses with 1-bit Time Reversal in a Solid Waveguide”, J. Acoust. Soc. Am., Vol.110, No.6, pp.2849-2857, (2001).

[4] Kim, J.S., and Shin, K.C., "ultiple Focusing with Adaptive Time-Reversal Mirror” J. Acoust. Soc. Am., Vol.115, No.2, pp.600-606, (2004).

[5] Miura, K., "Simple Calculation for SH Transient Wave Front Propagating through a Linear Gradient Inhomogeneous Half-Space " (in Japanese with English abstract), Transactions of JSME, Ser. A, Vol.67, No.663, pp.1742-1746, (2001).

[6] Miura, K., "Simulation of SH Transient Wave Reflection from a Micro Plane Crack in Elastic Medium” (in Japanese), Proceedings of the 2004 Annual Meeting of the JSME/MMD, pp.643-644, (2004).

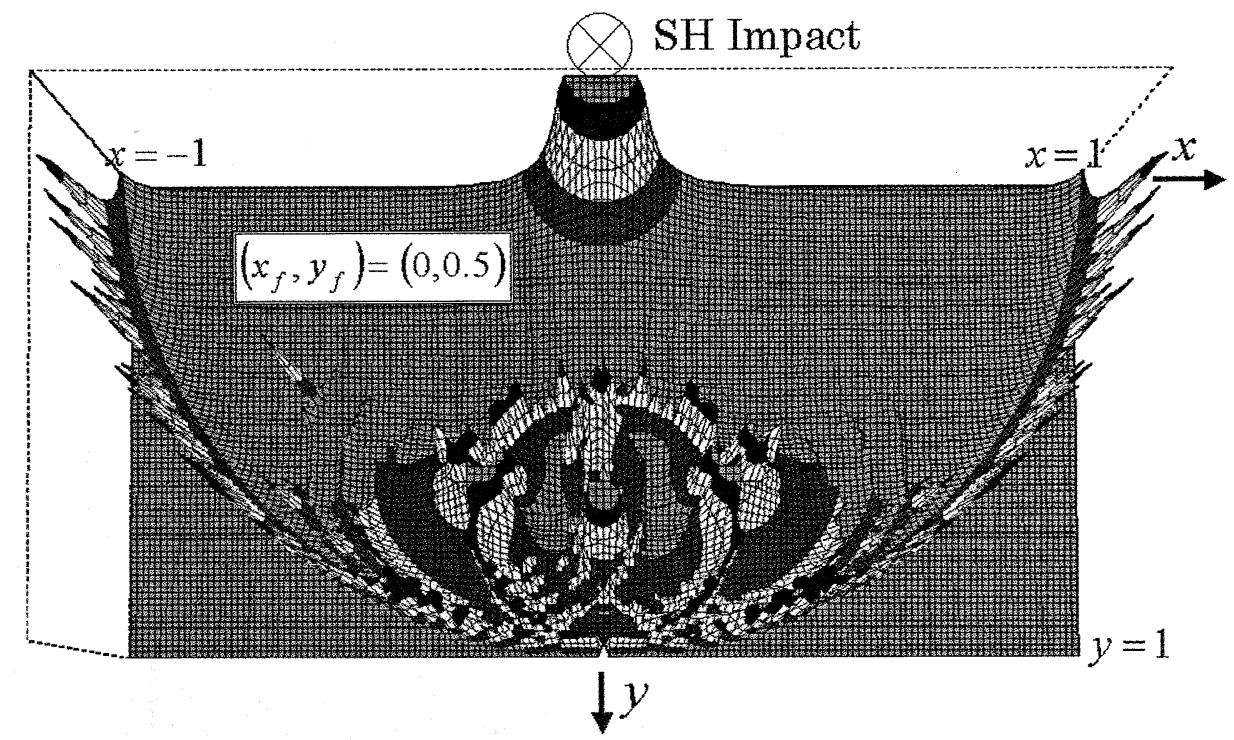

Figure 7 3-D graphic represent representation of the reflected strain energy from 9 micro plane cracks for the whole wave field 